\title{
Índice de calidad ecológica con base en macroinvertebrados acuáticos para la cuenca del río Negro (ICE $\left.\mathrm{RN}_{\mathrm{RAE}}\right)$, Colombia
}

Laura Cristina Forero ${ }^{1}$, Magnolia Longo ${ }^{1,2}$, John Jairo Ramírez R. ${ }^{1}$ \& Guillermo Chalar ${ }^{3}$

1. Grupo de Investigación en Limnología Básica y Experimental, Biología y Taxonomía Marina (LimnoBasE y Biotamar), Instituto de Biología, Universidad de Antioquia, Medellín, Colombia; lauracristinaforero@gmail.com, johnra77@gmail.com

2. Grupo de Limnología, Programa Ciencias Ambientales, Universidad de Bogotá Jorge Tadeo Lozano, Bogotá, Colombia; mc_longo@hotmail.com

3. Departamento de Limnología, Facultad de Ciencias, Universidad de la República, Montevideo, Uruguay; gchalar@fcien.edu.uy

Recibido 12-XII-2013. Corregido 20-I-2014. Aceptado 13-II-2014.

\begin{abstract}
Aquatic Ecological Index based on freshwater $\left(\mathrm{ICE}_{\mathrm{RN}-\mathrm{MAE}}\right)$ for the Río Negro watershed, Colombia. Available indices to assess the ecological status of rivers in Colombia are mostly based on subjective hypotheses about macroinvertebrate tolerance to pollution, which have important limitations. Here we present the application of a method to establish an index of ecological quality for lotic systems in Colombia. The index, based on macroinvertebrate abundance and physicochemical variables, was developed as an alternative to the BMWP-Col index. The method consists on determining an environmental gradient from correlations between physicochemical variables and abundance. The scores obtained in each sampling point are used in a standardized correlation for a model of weighted averages (WA). In the WA model abundances are also weighted to estimate the optimum and tolerance values of each taxon; using this information we estimated the index of ecological quality based also on macroinvertebrate $\left(\mathrm{ICE}_{\mathrm{RN}-\mathrm{MAE}}\right)$ abundance in each sampling site. Subsequently, we classified all sites using the index and concentrations of total phosphorus (TP) in a cluster analysis. Using TP and $\mathrm{ICE}_{\mathrm{RN}-\mathrm{MAE}}$, mean, maximum, minimum and standard deviation, we defined threshold values corresponding to three categories of ecological status: good, fair and critical. Rev. Biol. Trop. 62 (Suppl. 2): 233-247. Epub 2014 April 01.
\end{abstract}

Key words: ecological status index, BMWP-Col, macroinvertebrates, environmental gradient, tropical stream.

Uno de los índices bióticos más empleados para evaluar la calidad del agua en Colombia es el Biological Monitoring Working PartyColombia (BMWP-Col) modificado por Roldán (2003). A pesar de su amplia utilización, sus resultados en diversas regiones han permitido identificar algunos puntos débiles, entre los que figuran: (1) el empleo de datos cualitativos de presencia/ausencia, ignorando otros caracteres importantes como la abundancia y la biomasa de los organismos; (2) el haber sido desarrollado principalmente para la zona centro-oriental de Antioquia, la cual posee características geomorfológicas y climáticas diferentes a las de otras zonas ubicadas dentro de Antioquia y a lo largo del país; (3) la identificación de los organismos solo hasta familia, desconociéndose que los rangos de tolerancia de los taxones dentro una misma familia pueden variar (Bispo, Froehlich \& Oliveira, 2002; Bonada, Zamora-Muñoz, Rieradevall \& Prat, 2004; Tomanova \& Tedesco, 2007; Domínguez \& Fernández, 2009); y (4) el que las puntuaciones o niveles de tolerancia de los taxones individuales se fundamenta en el criterio de expertos, más que en el uso integrado de dicho criterio con una herramienta numérica, que permita establecer de manera objetiva y rigurosa 
los umbrales de adaptación de los organismos a las variables abióticas consideradas críticas (Hurford, Schneider \& Cowx, 2010).

Otras variantes del índice BMWP-Col han sido implementadas en diferentes regiones de Colombia (Zamora, 1999; Zúñiga \& Cardona, 2009); y adaptaciones del BMWP original de Armitage et al. (1983) han sido formulas en países de América Central (MINAE-SALUD, 2007; Springer, Vásquez, Castro \& Kohlmann, 2007) y del Sur (Medina, Hora, Pereda, Aguilar \& Guzmán, 2010), así como en el Caribe (Muñoz-Riveaux et al., 2003). Sin embargo, por las falencias mencionadas, las comunidades de investigadores de Colombia (Riss, Ospina \& Gutiérrez, 2002a; Gutiérrez, Riss \& Ospina, 2004) y otros países de Centro y Sur América, se han empeñado en elaborar índices que consideran criterios más rigurosos para evaluar la calidad del agua; tal es el caso de Moya, Tomanova \& Oberdorff (2007) en Bolivia, Acosta, Ríos, Rieradevall \& Prat (2009) en Ecuador y Perú (Índice de Calidad Ecológica de los Ríos Altoandinos: CERA); y Chalar, Arocena, Pacheco \& Fabián, (2011) en Uruguay (Trophic State Index for Benthic Invertebrates: TSI-BI). Sobre la importancia y los problemas relacionados con la elaboración de estos índices existe una buena cantidad de publicaciones teóricas y metódicas (Prat, Gonzáles, \& Millet, 1986; Alba-Tercedor \& Sánchez-Ortega, 1988; Hellawell, 1989; Rosenberg \& Resh, 1993; Alba-Tercedor, 1996; Diamond, Barbour, \& Stribling, 1996; ZamoraMuñoz \& Alba-Tercedor, 1996; Reynoldson, Norris, Resh, Day \& Rosenberg, 1997; Kelly \& Whitton, 1998).

Con el fin de mejorar los inconvenientes mencionados se presenta el desarrollo de un índice de calidad ecológica, generado a partir de los muestreos de macroinvertebrados y de variables fisicoquímicas realizados en la cuenca del río Negro ( $\mathrm{ICE}_{\mathrm{RN}-\mathrm{MAE}}$ ). Éste es formulado con base en análisis multivariados, tomando como referencia los métodos previamente descritos por Haase \& Nolte (2008) y Chalar et al. (2011). En él se propone un método para la elaboración de un sistema de bioindicación en el que la calidad ecológica se establece mediante: (1) un gradiente ambiental establecido a través de un análisis de correspondencia; (2) identificación para cada taxón, de rangos de tolerancia y valores óptimos en función de ese gradiente ambiental; y (3) ponderaciones de calidad para cada estación. La relevancia de este índice es que permite establecer cuantitativamente para los géneros, valores de tolerancia ante condiciones adversas; estos valores son identificados de acuerdo con el análisis integrado de las variables ambientales locales junto con la composición y abundancia de los organismos. El método de cálculo usado no está restringido a un sistema particular ni a un grupo de organismos específico. La herramienta fundamental para su elaboración es el 'monitoreo biológico', un instrumento centrado en el uso de los atributos estructurales $\mathrm{y} / \mathrm{o}$ funcionales de los individuos, las poblaciones, las comunidades y los ecosistemas (Hart, 1994). Dichos atributos, mediante un sistema de bioindicación pueden ser traducidos en un índice o valor indicador de una condición ecológica, permitiendo así una evaluación más adecuada de los ecosistemas considerando la variabilidad climática, edáfica, geomorfológica y biológica que ocurre dentro de las regiones tropicales.

\section{MATERIALES Y MÉTODOS}

Área de estudio: Este estudio se desarrolló en la cuenca del río Negro (Colombia), sometida a transformaciones espaciales de los ecosistemas acuáticos dado el aumento en los asentamientos industriales de los sectores textil, manufacturero y químico, además del incremento demográfico en la región (Roldán \& Pérez, 1978; Roldán, 1980; Arango \& Roldán, 1983; Acosta, 2005). Esta cuenca está ubicada al oriente de la cordillera central de Los Andes. Abarca un área de $952 \mathrm{~km}^{2}$ y presenta pequeñas colinas de cimas redondeadas donde nacen sus principales afluentes. La zona de vida predominante corresponde al bosque húmedo Montano-Bajo (Holdridge, 1982). La elevación del territorio varía entre los 2100 y los $2600 \mathrm{msnm}$, con precipitaciones promedios anuales que 
oscilan entre los 1800 y los 2500mm (Gaviria, 2000). Se presentan dos periodos de lluvia comprendidos entre los meses de abril-junio y septiembre-noviembre; siendo mayo y octubre los meses con mayores registros, mientras que en enero y febrero se presentan las menores precipitaciones (Toro, 1979). En la región existe un notable desarrollo económico sustentado en la floricultura, la agricultura y en industrias de diversos sectores. La disponibilidad de agua, las temperaturas constantes y la fertilidad del suelo han sido factores relevantes para el desarrollo agropecuario, por tanto el paisaje ha presentado transformaciones relevantes en cuanto a sus componentes y estados de los mismos (Machado \& Ramírez, 2003).

Campañas de muestreo: En el 2002 se realizaron dos muestreos, uno en marzo y otro en junio (periodo de lluvias escasas). Se muestrearon en total 19 estaciones, distribuidas entre siete quebradas (afluentes principales del río) y en el eje longitudinal del río (Cuadro 1). En cada estación se delimitó un tramo de $50 \mathrm{~m}$ de longitud dentro del cual se recolectaron en diferentes tipos de sustrato las muestras de macroinvertebrados, comenzando desde la parte baja. Los sustratos más representativos incluyeron gravas, cantos, vegetación ribereña, macrófitas y lodos. Para muestrear el sustrato pedregoso se utilizó una red Surber, para la vegetación ribereña una tipo $\mathrm{D}$ con una abertura de malla de $500 \mu \mathrm{m}$, y para los sustratos lodosos una draga tipo Ekman. En los sustratos disponibles y usando el aparejo indicado, se realizaron en total diez arrastres por estación, cada uno con una duración de un minuto. Las diez muestras provenientes de los diferentes tipos de sustrato presentes en cada estación, se unificaron en una sola. Las abundancias de

\section{CUADRO 1}

Coordenadas geográficas, elevación sobre el nivel del mar y actividades antrópicas asociadas a cada estación de muestreo en la cuenca del río Negro

TABLE 1

Geographical coordinates, elevation above sea level and human activities associated to each sampling station in Negro river watershed

\begin{tabular}{|c|c|c|c|c|c|}
\hline Cuerpo de agua & Código & Estación & Coordenadas & $\begin{array}{c}\text { Elevación } \\
\text { msnm }\end{array}$ & $\begin{array}{l}\text { Actividad } \\
\text { humana }\end{array}$ \\
\hline Quebrada & E1 & Sector La Feria & $06^{\circ} 07^{\prime} 56^{\prime \prime} \mathrm{N}-75^{\circ} 15^{\prime} 31^{\prime \prime} \mathrm{W}$ & 2137 & Agricultura \\
\hline \multirow[t]{2}{*}{ La Marinilla } & E2 & La Amistad & $06^{\circ} 08^{\prime} 12^{\prime \prime} \mathrm{N}-75^{\circ} 16^{\prime} 10^{\prime \prime} \mathrm{W}$ & 2135 & Industria \\
\hline & E3 & Bomba El Cordobés & $06^{\circ} 11^{\prime} 07^{\prime \prime} \mathrm{N}-75^{\circ} 21^{\prime} 02^{\prime \prime} \mathrm{W}$ & 2092 & Industria Agricultura \\
\hline \multirow[t]{4}{*}{ Río Negro } & $\mathrm{E} 4$ & El Hierbal & $06^{\circ} 07^{\prime} 49^{\prime \prime} \mathrm{N}-75^{\circ} 30^{\prime} 18^{\prime \prime} \mathrm{W}$ & 2279 & Ninguna \\
\hline & E5 & Montenevado & $06^{\circ} 07^{\prime} 02^{\prime \prime} \mathrm{N}-75^{\circ} 30^{\prime} 03^{\prime \prime} \mathrm{W}$ & 2182 & Ninguna \\
\hline & E6 & Puente Real & $06^{\circ} 08^{\prime} 34^{\prime \prime} \mathrm{N}-75^{\circ} 22^{\prime} 49^{\prime \prime} \mathrm{W}$ & 2104 & Industria \\
\hline & E7 & Puente Autopista & $06^{\circ} 10^{\prime} 45^{\prime \prime} \mathrm{N}-75^{\circ} 21^{\prime} 42^{\prime \prime} \mathrm{W}$ & 2094 & Industria \\
\hline Quebrada & E8 & Puente Betania & $06^{\circ} 04^{\prime} 36^{\prime \prime} \mathrm{N}-75^{\circ} 19^{\prime} 51^{\prime \prime} \mathrm{W}$ & 2170 & Industria Agricultura \\
\hline \multirow[t]{2}{*}{ La Cimarrona } & E9 & Flor Silvestre & $06^{\circ} 05^{\prime} 51^{\prime \prime} \mathrm{N}-75^{\circ} 20^{\prime} 28^{\prime \prime} \mathrm{W}$ & 2178 & Agricultura \\
\hline & E10 & Textiles Río Negro & $06^{\circ} 10^{\prime} 08^{\prime \prime} \mathrm{N}-75^{\circ} 21^{\prime} 26^{\prime \prime} \mathrm{W}$ & 2099 & Industria \\
\hline \multirow[t]{3}{*}{ Quebrada La Pereira } & E11 & Divino Niño & $06^{\circ} 01^{\prime} 19^{\prime \prime} \mathrm{N}-75^{\circ} 25^{\prime} 52^{\prime \prime} \mathrm{W}$ & 2158 & Agricultura \\
\hline & E12 & Puente San Sebastián & $06^{\circ} 02^{\prime} 26^{\prime \prime} \mathrm{N}-75^{\circ} 24^{\prime} 59^{\prime \prime} \mathrm{W}$ & 2142 & Floricultivo \\
\hline & E13 & Puente Comfama & $06^{\circ} 08^{\prime} 26^{\prime \prime} \mathrm{N}-75^{\circ} 22^{\prime} 53^{\prime \prime} \mathrm{W}$ & 2102 & Industria \\
\hline Quebrada Chachafruto & E14 & Puente Aeropuerto & $06^{\circ} 09^{\prime} 43^{\prime \prime} \mathrm{N}-75^{\circ} 24^{\prime} 55^{\prime \prime} \mathrm{W}$ & 2116 & Industria \\
\hline \multirow[t]{2}{*}{ Quebrada El Salado } & E15 & Campamento & $06^{\circ} 17^{\prime} 10^{\prime \prime} \mathrm{N}-75^{\circ} 20^{\prime} 05^{\prime \prime} \mathrm{W}$ & 2159 & Agricultura \\
\hline & E16 & La Compañía & $06^{\circ} 15^{\prime} 31^{\prime \prime} \mathrm{N}-75^{\circ} 20^{\prime} 51^{\prime \prime} \mathrm{W}$ & 2135 & Agricultura \\
\hline \multirow[t]{3}{*}{ Quebrada La Mosca } & E17 & El Molino & $06^{\circ} 18^{\prime} 25^{\prime \prime} \mathrm{N}-75^{\circ} 27^{\prime} 32^{\prime \prime} \mathrm{W}$ & 2183 & Ninguna \\
\hline & E18 & Gases de Antioquia & $06^{\circ} 15^{\prime} 57^{\prime \prime} \mathrm{N}-75^{\circ} 26^{\prime} 16^{\prime \prime} \mathrm{W}$ & 2144 & Industria Agricultura \\
\hline & E19 & RIOTEX & $06^{\circ} 10^{\prime} 53^{\prime \prime} \mathrm{N}-75^{\circ} 21^{\prime} 59^{\prime \prime} \mathrm{W}$ & 2095 & Industria \\
\hline
\end{tabular}


los organismos se reportaron en número de individuos por esfuerzo de muestreo (10min). Los organismos obtenidos fueron almacenados en frascos plásticos con alcohol etílico al 96\%.

Los organismos fueron separados $\mathrm{y}$ cuantificados por género y morfotipo, y las abundancias registradas en marzo y junio se promediaron. Para evitar sesgos en la elaboración del índice a causa de los taxones con baja abundancia y/o frecuencia, se eliminaron de los análisis los grupos que se encontraron en una sola estación y aquellos con abundancias menores o iguales a cinco individuos, según Resh, Bêche \& McElravy (2005). Para las identificaciones se usaron las claves taxonómicas de Roldán (1988), Smith (1989), Schuh \& Slater (1995), Wiggins (1996), Milligan (1997), Epler (2001), Aristizábal (2002), Carvalho (2002), Navarrete-Heredia, Newton \& Thayer (2002), Martínez (2005), Bejarano (2006), Dominguez et al. (2006), Heckman (2006; 2008), Borkent \& Spinelli (2007), Domínguez \& Fernández (2009) y Tejerina \& Paggi (2010).
En el Cuadro 2 se resumen las variables fisicoquímicas registradas en la cuenca del río Negro. In situ se midieron las variables: $\mathrm{pH}$, oxígeno disuelto, conductividad, temperatura del agua y color. Para la determinación de las concentraciones de nutrientes y de las demandas química y biológica de oxígeno, se almacenaron muestras de agua de cada sitio en recipientes plásticos de $500 \mathrm{~mL}$, las cuales fueron conservadas a una temperatura mayor a cinco y menor a $10^{\circ} \mathrm{C}$; estas muestras fueron procesadas en el Centro de Servicios de Análisis de Aguas de CORNARE (CENSA).

Análisis estadístico: El método empleado para la elaboración del índice de calidad ecológica ( $\left.\mathrm{ICE}_{\mathrm{RN}-\mathrm{MAE}}\right)$ está basado en la propuesta de Chalar et al. (2011). Esta consiste en cinco pasos: (1) Correlaciones de Spearman entre las variables fisicoquímicas para eliminar variables redundantes. (2) Determinación de un gradiente ambiental desde un análisis lineal (análisis de redundancia sin tendencia,

CUADRO 2

Variables físicoquímicas registradas en las estaciones de la cuenca del río Negro, unidades, equipos y métodos de medición empleados

TABLE 2

Physicochemical variables recorded in the Negro river watershed, units, equipment and/or measurement methods employed

\begin{tabular}{|c|c|c|}
\hline Variable & Unidad & Equipo y/o método \\
\hline $\mathrm{pH}$ & Unidades de $\mathrm{pH}$ & $\mathrm{pH}$-metro \\
\hline Temperatura de agua & ${ }^{\circ} \mathrm{C}$ & Termómetro \\
\hline Oxígeno disuelto & $\mathrm{mg} / \mathrm{L} \mathrm{O}_{2}$ & Oxímetro \\
\hline Demanda bioquímica de oxígeno & $\mathrm{mg} / \mathrm{L} \mathrm{O}_{2}$ & Oxímetro/potenciométrico, Prueba de los 5 días \\
\hline Sólidos suspendidos totales & $\mathrm{mg} / \mathrm{L} \mathrm{SST}$ & Gravimétrico/secado $(103-105)^{\circ} \mathrm{C}$ \\
\hline Sólidos disueltos totales & $\mathrm{mg} / \mathrm{L} \mathrm{SDT}$ & Gravimétrico/cálculo por diferencia \\
\hline Conductividad eléctrica & $\mu \mathrm{S} / \mathrm{cm}$ & Conductímetro/electrométrico \\
\hline Fósforo total & $\mu \mathrm{g} / \mathrm{L}$ & Colorimétrico/ácido ascórbico \\
\hline Nitratos & $\mathrm{mg} / \mathrm{L} \mathrm{NO}_{3-}$ & Espectrométrico ultravioleta selectivo (4 500- $\left.\mathrm{NO}_{3-} \mathrm{B}\right)$ \\
\hline Nitritos & $\mathrm{mg} / \mathrm{L} \mathrm{NO}_{2}$ & Espectrométrico ultravioleta selectivo (4 500- $\left.\mathrm{NO}_{3-} \mathrm{B}\right)$ \\
\hline Nitrógeno amoniacal & $\mathrm{mg} / \mathrm{L} \mathrm{NH}_{3}-\mathrm{N}$ & Titulométrico/destilación \\
\hline Nitrógeno total Kjeldahl & $\mathrm{mg} / \mathrm{L} \mathrm{N}$ & Titulométrico/micro Kjeldahl \\
\hline Sulfatos & $\mathrm{mg} / \mathrm{L} \mathrm{SO}_{4}^{-2}$ & Turbidimétrico $\left(45000-\mathrm{SO}_{4}{ }^{-2}\right)$ \\
\hline Alcalinidad total & $\mathrm{mg} / \mathrm{L} \mathrm{CaCO}_{3}$ & Titulométrico (2 320-B) \\
\hline Color & U.C. & Disco Secchi \\
\hline Turbiedad & N.T.U. & Nefelométrico (2 130-B) \\
\hline
\end{tabular}


RDA por sus siglas en inglés) o uno unimodal (Análisis de Correspondencia Canónica, ACC), según sea el caso, utilizando el programa CANOCO versión 4.5 (Ter Braak \& Smilauer, 2002). Se utilizaron los datos medios de las abundancias y las medias de los registros de las variables fisicoquímicas. Para explicar el gradiente ambiental se examinaron además, las correlaciones de Spearman entre las variables significantes y las no significantes en el ACC o RDA. (3) Estandarización de los puntajes de las estaciones (sample scores) obtenidos desde el primer eje del RDA o del ACC. Ésta consiste en re-dimensionar los datos a una nueva escala entre uno y diez utilizando una regresión lineal. La regresión es formulada desde los valores máximo y mínimo de los sample scores. (4) En el programa Análisis de Datos C2 (software libre) se obtuvieron los puntajes óptimos y de tolerancia para cada taxón, a partir de un análisis de Promedios Ponderados (Weighted Averaging, WA) utilizando los datos re-escalados. Con estos puntajes se calcularon los valores del índice de estado ecológico por estación, con base en la fórmula propuesta por Haase \& Nolte (2008). (5) Agrupamiento de las estaciones a partir de los valores del índice de estado ecológico junto con los datos de la variable fisicoquímica indicadora (p.e. fósforo total, Nitratos). Con ello se obtuvieron los rangos para establecer las categorías del índice. Para este análisis se empleó como método de agrupamiento los pares de grupos no ponderados aplicando la media aritmética (UPGMA, por sus siglas en inglés) y la distancia euclidiana.

De acuerdo con la longitud del gradiente del primer eje (2.16 desviación estándar) obtenido en el análisis de correspondencia sin tendencia (DCA, por sus siglas en inglés), se identificó que el método de ordenación más robusto para establecer el gradiente ambiental era un análisis unimodal (ACC). En este caso se realizaron varios ACC puesto que desde el principio se encontró que el OD era una variable altamente explicativa y que por tanto enmascaraba a otras variables relevantes. Así, en el primer ACC se encontró que el OD explicaba un $16.09 \%$ del $25.13 \%$ de la varianza total; por tanto, se decidió ejecutar otro ACC colocando esta variable como suplementaria para determinar si existían otras variables explicativas significantes. Se tomó esta decisión debido a que el OD en este tipo de índices no es un buen indicador puesto que en la zona tropical fluctúa en función de otras variables y/o circunstancias, tales como, cambios diurnos y nocturnos, temperatura hídrica, descargas de desechos orgánicos e inorgánicos y movimiento del agua en función de las lluvias y los vientos, entre otras (Dudgeon, 2008). Adicionalmente, mediante un análisis de correlación de Spearman se estableció el grado de asociación entre los resultados del $\operatorname{ICE}_{\mathrm{RN}-\mathrm{MAE}} \mathrm{y}$ el BMWP-Col. Los datos fueron estandarizados previamente siguiendo el método propuesto por Guisande-González et al. (2006).

\section{RESULTADOS}

Del total de géneros y morfotipos, que fueron 97 en los dos muestreos, se emplearon en el análisis solo aquellos presentes en más de una estación de muestreo, es decir 58 taxones. El número medio de géneros y morfotipos en los sitios fue 21 con un valor mínimo de siete y un máximo de 46 . La abundancia media fue de $100 \pm 132$ individuos.

El resultado de las correlaciones de Spearman para las variables fisicoquímicas, mostró una alta asociación $(\mathrm{r} \geq 0.60)$ entre la DQO, los ortofosfatos y los sólidos totales con el fósforo total; mientras que los sólidos disueltos, la $\mathrm{DBO}$, el nitrógeno total y fueron relacionados al oxígeno disuelto, por lo que el primer grupo de variables fue eliminado de análisis posteriores.

\section{Determinación del gradiente ambiental:} En el ACC, el primer eje presentó el eigenvalue más alto $(0.22)$, lo que indica un fuerte gradiente de variación. Los ejes dos, tres y cuatro mostraron valores de $0.10,0.16$ y 0.15 , respectivamente. La inercia total de los taxones fue de 1.40, de la cual el primer eje explicó el $15.9 \%$ de la variación, y los tres restantes entre el 6.9 y $12.9 \%$. 
De las variables fisicoquímicas, el fósforo total más la temperatura explicaron 0.32 $(22.7 \%)$ del total de la varianza. El fósforo tuvo una alta correlación positiva con el primer eje (0.89), mientras que la temperatura se relacionó negativamente con el eje dos (-0.71).

Dado que el fósforo total estuvo significativa y positivamente correlacionado (Spearman: $n=18, r>0.60, p<0.05$ ) con los ortofosfatos, la conductividad, las concentraciones de nitrógeno total y amoniacal, y los sulfatos; y negativamente con el oxígeno disuelto, el primer eje del ACC representó un gradiente de enriquecimiento por nutrientes provenientes de las actividades industrial y agropecuaria. A este eje se asociaron por tanto las estaciones con los mayores valores de fósforo total pertenecientes al grupo uno de la Fig. 1. Por otro lado, la temperatura, la cual estuvo relacionada con el eje dos (Spearman: $\mathrm{n}=18, \mathrm{r}>60, \mathrm{p}<0.05)$, se asoció significativamente con los sólidos suspendidos y disueltos, el color y la turbidez, lo que indica un gradiente de disminución en la penetración de los rayos solares en la columna de agua. Las estaciones de los grupos dos y tres de la Fig. 1, estuvieron asociadas con este eje.

En resumen, el cambio en las condiciones fisicoquímicas de los sitios estudiados queda expresado en el ordenamiento de los mismos a lo largo de los ejes. De esta manera, los puntajes de las estaciones en el ACC (sample scores) representan objetivamente su posición en el gradiente de variación ambiental y son la base para calcular el índice de calidad ecológica.

El re-escalonamiento de los puntajes de las estaciones obtenidos en el ACC se realizó mediante la ecuación $\mathrm{y}=7.69-4.80 \mathrm{X}$, donde $\mathrm{X}$ se reemplazó con el valor sample scores de cada estación. Las puntuaciones fueron redimensionadas a una escala entre uno y diez, donde uno representa aguas contaminadas y diez aguas limpias.

Valores óptimos y de tolerancia de cada taxón: Estos valores se obtuvieron desde un modelo de promedios ponderados, que en este

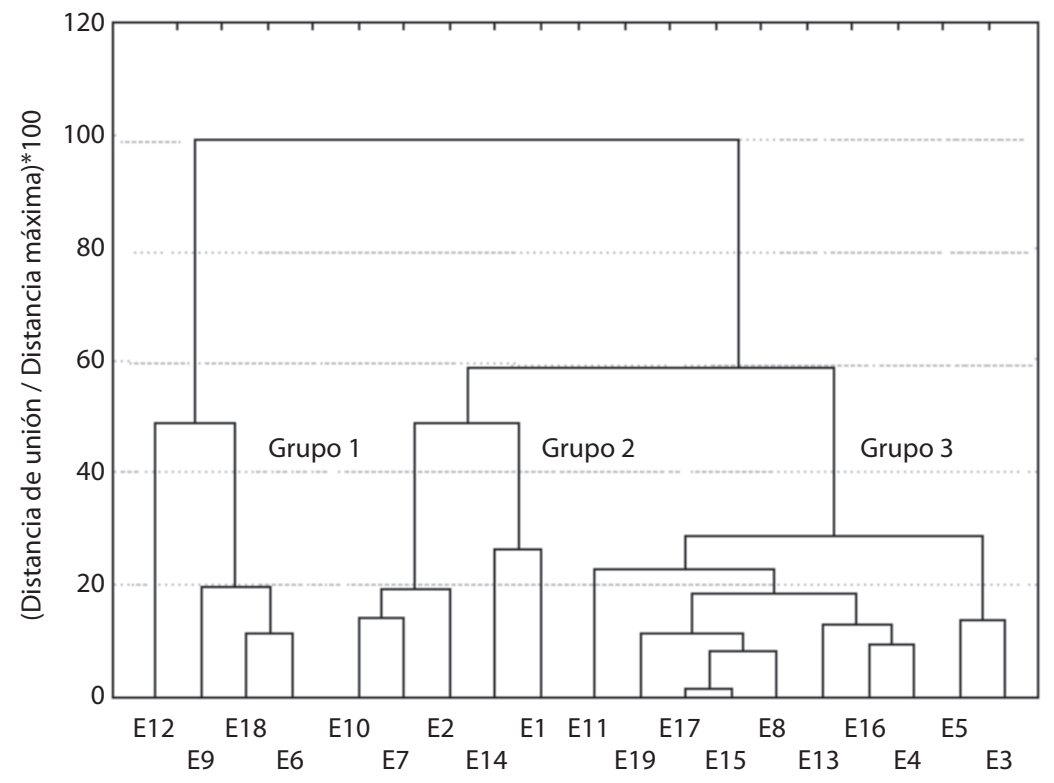

Fig. 1. Agrupamiento de las estaciones de acuerdo con los puntajes obtenidos del Índice de Calidad Ecológica con base en macroinvertebrados acuáticos para la cuenca del río Negro ( $\mathrm{ICE}_{\mathrm{RN}-\mathrm{MAE}}$ ) y las concentraciones de fósforo total en cada estación.

Fig. 1. Sampling stations grouped according to the scores of the Ecological Quality Index based on macroinvertebrates for the Negro river watershed ( $\mathrm{ICE}_{\mathrm{RN}-\mathrm{MAE}}$ ) and total phosphorus concentrations at each station. 
caso contó con un alto coeficiente de determinación $\left(\mathrm{r}^{2}=0.80\right)$ y un bajo error cuadrático medio $(\mathrm{ECM}=1.33)$. El valor óptimo $\left(\mathrm{Op}_{\mathrm{i}}\right)$ corresponde al puntaje de cada taxón en el gradiente de variación ambiental, que va de aguas afectadas por aportes orgánicos e inorgánicos a aguas limpias. La tolerancia (Tol) describe la amplitud ecológica a lo largo del gradiente ambiental. Cincuenta y un taxones presentaron puntuaciones óptimas asociadas a condiciones de buena calidad ecológica $\left(\mathrm{Op}_{\mathrm{i}}\right.$ $>6.1$, Cuadro 5), cuatro estuvieron relacionados con una situación regular $\left(\mathrm{Op}_{\mathrm{i}}=5-6\right)$, y tres presentaron valores correspondientes a estados de calidad crítica $\left(\mathrm{Op}_{\mathrm{i}}<4.9\right)$ (Cuadro 3).

Estos valores junto con los de abundancia $\left(A b_{i}\right)$, se utilizaron para calcular el índice de estado ecológico mediante la aplicación de la fórmula propuesta por Haase \& Nolte (2008): $\mathrm{ICE}_{\mathrm{RN}-\mathrm{MAE}}=\left(\sum \mathrm{Op}_{\mathrm{i}} * \mathrm{Tol}_{\mathrm{i}} * \mathrm{Ab}_{\mathrm{i}}\right) /($ $\left(\sum \mathrm{Op}_{\mathrm{i}}{ }^{*} \mathrm{Tol}_{\mathrm{i}} * \mathrm{Ab}_{\mathrm{i}}\right)$.

Índice: Los valores del $\mathrm{ICE}_{\mathrm{RN}-\mathrm{MAE}}$ para cada estación fluctuaron entre 3.4 y 8.1. Estos valores están alta y significativamente correlacionados con las puntuaciones de las muestras

CUADRO 3

Macroinvertebrados bénticos encontrados en la cuenca del río Negro con los correspondientes registros óptimos $\left(\mathrm{Op}_{\mathrm{i}}\right)$ y de tolerancia (Tol) asociados a condiciones fisicoquímicas

TABLE 3

Benthic macroinvertebrates found in the Negro river watershed with the corresponding optimal $\left(\mathrm{Op}_{\mathrm{i}}\right)$ and tolerance (Tol) scores associated to physicochemical conditions

\begin{tabular}{|c|c|c|c|c|}
\hline Orden & Familia & Género & $\mathrm{Op}_{\mathrm{i}}$ & Tol \\
\hline Haplotaxida & Indeterminado & sp. & 1.7 & 3.4 \\
\hline Rhynchobdellida & Glossiphonidae & Hellobdella & 5.3 & 2.3 \\
\hline Pharyngobdellida & Erpobdellidae & sp. & 7.0 & 2.6 \\
\hline \multirow[t]{4}{*}{ Basommatophora } & Ancylidae & sp. & 8.6 & 2.1 \\
\hline & Lymnaeidae & Lymnaea & 9.2 & 0.5 \\
\hline & Physidae & Physa & 9.4 & 1.5 \\
\hline & Planorbidae & Biomphalaria & 9,9 & 0.4 \\
\hline Mesogastropoda & Hydrobiidae & sp. & 8.5 & 2.1 \\
\hline Veneroida & Sphaeriidae & sp. & 8.9 & 1.6 \\
\hline Hydracarina & Indeterminado & sp. & 7.8 & 1.8 \\
\hline \multirow[t]{3}{*}{ Ephemeroptera } & Baetidae & Americabaetis & 9.1 & 0.7 \\
\hline & & Baetodes & 9.0 & 1.4 \\
\hline & & Camelobaetidius & 9.8 & 0.1 \\
\hline \multirow[t]{6}{*}{ Odonata } & Calopterygidae & Hetaerina & 9.6 & 0.7 \\
\hline & Coenagrionidae & Argia & 8.0 & 1.6 \\
\hline & Libellulidae & Brechmorhoga & 9.0 & 1.2 \\
\hline & & Erythemis & 8.2 & 1.2 \\
\hline & Gomphidae & Progomphus & 9.7 & 0.4 \\
\hline & Aeshnidae & Rhionaeschna & 8.3 & 1.0 \\
\hline \multirow[t]{3}{*}{ Hemiptera } & Mesoveliidae & Mesoveloidea & 9.7 & 0.1 \\
\hline & Naucoridae & Limnocoris & 9.7 & 0.1 \\
\hline & Veliidae & Rhagovelia & 8.9 & 0.8 \\
\hline \multirow[t]{5}{*}{ Coleoptera } & Elmidae & Heterelmis & 9.6 & 0.1 \\
\hline & Gyrinidae & Gyrinus & 6.4 & 2.2 \\
\hline & Hydrophylidae & sp. & 6.2 & 3.5 \\
\hline & Ptilodactylidae & Tetraglossa & 9.8 & 0.1 \\
\hline & Staphylinidae & sp. & 6.2 & 2.3 \\
\hline
\end{tabular}


CUADRO 3 (Continuación) / TABLE 3 (Continued)

\begin{tabular}{|c|c|c|c|c|}
\hline Orden & Familia & Género & $\mathrm{Op}_{\mathrm{i}}$ & Tol \\
\hline \multirow[t]{8}{*}{ Trichoptera } & Calamoceratidae & Phylloicus & 8.9 & 0.9 \\
\hline & Glossosomatidae & Culoptila & 9.7 & 0.3 \\
\hline & Helichopsychidae & Helichopsyche & 9.5 & 1.0 \\
\hline & Hydrobiosidae & Atopsyche & 9.6 & 0.6 \\
\hline & Hydropsychidae & Leptonema & 9.7 & 0.1 \\
\hline & Hydroptilidae & Metrichia & 9.5 & 0.6 \\
\hline & & Oxyethira & 8.8 & 1.4 \\
\hline & Leptoceridae & Nectopsyche & 9.6 & 0.5 \\
\hline Lepidoptera & Crambidae & sp. & 8.9 & 2.6 \\
\hline Megaloptera & Corydalidae & Corydalus & 7.4 & 1.4 \\
\hline \multirow[t]{21}{*}{ Diptera } & Chironomidae & Chironomus & 3.7 & 1.2 \\
\hline & & Polypedylum & 7.4 & 2.9 \\
\hline & & Orthocladiinae sp. 1 & 5.2 & 2.7 \\
\hline & & Orthocladiinae sp. 2 & 9.5 & 0.9 \\
\hline & & Parachironomus & 7.1 & 1.6 \\
\hline & & Pseudochironomus & 8.4 & 1.6 \\
\hline & & Paratanytarsus & 8.6 & 1.6 \\
\hline & & Endotribelos & 9.4 & 0,7 \\
\hline & & Tanypodinae sp. 1 & 8.1 & 3.8 \\
\hline & Empididae & Chelifera & 9.8 & 0.1 \\
\hline & & Hemerodromia & 9.7 & 0.1 \\
\hline & Ephydridae & sp. 1 & 9.8 & 1.4 \\
\hline & & sp. 2 & 7.1 & 1.4 \\
\hline & Muscidae & Lispe & 2.2 & 1.7 \\
\hline & Psychodidae & Clognia & 6.2 & 2.2 \\
\hline & & Psychoda & 5.9 & 1.9 \\
\hline & Simuliidae & Simulium & 9.6 & 0.6 \\
\hline & Syrphidae & Eristalis & 8.3 & 2.2 \\
\hline & Tipulidae & Limonia & 6.9 & 1.2 \\
\hline & & Molophilus & 6.4 & 2.1 \\
\hline & & Tipula & 9.6 & 0.3 \\
\hline
\end{tabular}

en el primer eje del ACC (Spearman: $\mathrm{r}=-0.88$, $\mathrm{p}<0.05$ ); lo que significa que el índice resume la mayoría de la información referente a la distribución de los macroinvertebrados con respecto al gradiente de variación ambiental.

Para establecer grupos de estaciones con condiciones ecológicas similares, se realizó un análisis de agrupamiento con base en los datos del $\mathrm{ICE}_{\mathrm{RN}-\mathrm{MAE}} \mathrm{y}$ de la variable indicadora. La elección de esta variable es tal vez el punto más crítico para la implementación del índice, pues para definirla se requiere no sólo de las pruebas estadísticas que podrían ayudar a identificarla sino también del conocimiento del investigador acerca de las condiciones que debe cumplir una variable para ser considerada como indicadora (ver definición y características en Bakkes, 1994). En este caso se eligió al fósforo total como indicador puesto que, constituye un elemento aceptado ampliamente como limitante para la producción primaria. Además, el fósforo es relativamente estable durante el día, por lo que no presenta cambios bruscos en su concentración, como sí ocurre con el oxígeno disuelto.

En el agrupamiento se obtuvieron tres grupos significativamente diferentes (T-test: $\mathrm{p}<0.05$, Fig. 1). El grupo uno reunió estaciones con el mayor valor medio de fósforo total y el 
CUADRO 4

Valores medios, mínimos, máximos y Desviación Estándar (DE) de los puntajes de ICE $_{\mathrm{RN}-\mathrm{MAE}}$ $\mathrm{y}$ de los registros de fósforo total para los tres grupos obtenidos desde el análisis de agrupamiento

TABLE 4

Mean, minimum, and maximum values, and standard deviation (DE) of ICE $\mathrm{RN}_{\mathrm{RAE}}$ scores and of the total phosphorus concentrations for three groups defined by cluster

\begin{tabular}{lcccccc}
\multicolumn{2}{c}{ Estimativos } & \multicolumn{2}{c}{ Grupo 1} & \multicolumn{2}{c}{ Grupo 2 } & \multicolumn{2}{c}{ Grupo 3} \\
Media & $\mathrm{FT}(\mu \mathrm{g} / \mathrm{L})$ & $\mathrm{ICE}_{\mathrm{RN}-\mathrm{MAE}}$ & $\mathrm{FT}(\mu \mathrm{g} / \mathrm{L})$ & $\mathrm{ICE}_{\mathrm{RN}-\mathrm{MAE}}$ & $\mathrm{FT}(\mu \mathrm{g} / \mathrm{L})$ & $\mathrm{ICE}_{\mathrm{RN}-\mathrm{MAE}}$ \\
Mínimo & 359 & 5 & 159 & 6 & 68 & 7 \\
Máximo & 339 & 3 & 48 & 5 & 27 & 6 \\
DE & 398 & 6 & 246 & 6 & 111 & 8 \\
\hline
\end{tabular}

mínimo de $\mathrm{ICE}_{\mathrm{RN}-\mathrm{MAE}}$. $\mathrm{El}$ grupo tres agrupó los sitios que tenían el mínimo valor medio de fósforo total y el máximo del índice. Y los sitios en el grupo dos mostraron valores intermedios para ambas variables (Cuadro 4). Estos resultados se corresponden con el encontrado en el ACC y, por tanto, se corrobora que las estaciones se agrupan siguiendo un gradiente que va de aguas con concentraciones altas de fósforo (grupo 1) a aguas con bajas concentraciones (grupo 3).

Posteriormente, los rangos de calidad ecológica se obtienen para dos variables, el fósforo total (variable indicadora) y el ICE $\mathrm{RN}_{\text {RAE. }}$ Para la definición de los rangos se utilizan los valores máximos y mínimos reportados en la Tabla 4. Así, para el fósforo los valores máximos de los grupos dos y tres $(246 \mu \mathrm{g} / \mathrm{L}$ y $111 \mu \mathrm{g} / \mathrm{L}$ respectivamente) sirvieron como valores umbrales de calidad (Cuadro 5). No obstante, cuando los valores máximos y mínimos se sobreponen se recomienda utilizar como límites los valores medianos, tal como se hizo para el $\mathrm{ICE}_{\mathrm{RN}-\mathrm{MAE}}$ (Cuadro 4).

Finalmente, al aplicar el índice, la calidad ecológica puede ser establecida con base en la medición de las concentraciones de fósforo total $(\mu \mathrm{g} / \mathrm{L})$ o con base en el cálculo del $\mathrm{ICE}_{\mathrm{RN}-\mathrm{MAE}}$ siguiendo los puntajes reportados en el Cuadro 3 y la fórmula de Haase \& Nolte (2008). Los resultados obtenidos se contrarrestan con las categorías presentadas en el Cuadro 5 y así se define la calidad. Como una manera de poner a prueba la clasificación de calidad ecológica propuesta y presentada en el Cuadro 5, se efectuó un análisis de correlación entre las puntuaciones asignadas a cada estación con el $\mathrm{ICE}_{\mathrm{RN}-\mathrm{MAE}} \mathrm{y}$ del BMWP-Col.

En la escala del BMWP-Col, un puntaje menor a diez corresponde a una calidad del agua muy crítica, y puntajes iguales o mayores a 100 representan calidad del agua buena. Valores intermedios comprenden categorías de calidad entre crítica y aceptable.

\section{CUADRO 5}

Valores umbrales de fósforo total $(\mu \mathrm{g} / \mathrm{L})$ y de $\mathrm{ICE}_{\mathrm{RN}-\mathrm{MAE}}$ que definen las categorías de calidad ecológica: buena, regular y crítica

TABLE 5

Total phosphorus $(\mu \mathrm{g} / \mathrm{L})$ and $\mathrm{ICE}_{\mathrm{RN}-\mathrm{MAE}}$ threshold values defining ecological quality classes: good, acceptable and critical

\begin{tabular}{lccc} 
& Grupo 1 & Grupo 2 & Grupo 3 \\
Calidad ecológica & Crítica & Regular & Buena \\
Fósforo total $(\mu \mathrm{g} / \mathrm{l})$ & $>246$ & $111-246$ & $<111$ \\
$\mathrm{ICE}_{\mathrm{RN}-\mathrm{MAE}}$ & $\leq 4.9$ & $5-6$ & $>6.1$ \\
\hline
\end{tabular}


De manera similar, la escala del ICE $_{\text {RN-MAE }}$ abarca un rango entre uno y diez. El número de categorías de calidad en ambos índices varía, conteniendo el BMWP-Col cinco y el $\mathrm{ICE}_{\mathrm{RN}}$ MAE tres (Cuadro 6). Esta situación conduce a que la equivalencia en ambos no sea apreciable en términos conceptuales para categorías como "aceptable", "dudosa" o "muy crítica", pero sí en términos numéricos, ya que su correlación es alta y significativa (Spearman: $\mathrm{r}=0.78, \mathrm{p}<0.05$ ).

\section{DISCUSIÓN}

Determinación del gradiente ambiental: Según se mencionó, las variables que definieron el gradiente ambiental en la cuenca estudiada fueron la temperatura y el fósforo total, ambas negativa y significativamente correlacionadas con el oxígeno disuelto. La causalidad de estas relaciones inversas está fundamentada en el metabolismo biológico que tiene lugar en los ecosistemas acuáticos, el cual está a su vez influenciado por las altas temperaturas tropicales; así, a mayor temperatura menor solubilidad de oxígeno y aumento de su demanda biológica dado el incremento en la tasa de oxidación (Esteves, 2011). Por su parte, al disminuir el oxígeno, el fósforo se solubiliza e incrementa su concentración en el agua, ocasionando la eutrofización del sistema y la consecuente disminución del oxígeno (Roldán \& Ramírez, 2008).

También se mencionó que el fósforo total está positivamente correlacionado con los sólidos disueltos y suspendidos, con las demandas química y bioquímica de oxígeno, con el nitrógeno total, la alcalinidad y la conductividad, mientras que el oxígeno disuelto esta negativamente correlacionado con estos. Estas relaciones indican que la entrada de materia orgánica e inorgánica a los sistemas está agotando la concentración de oxígeno. En consecuencia,

CUADRO 6

Clasificación del estado ecológico de las estaciones sobre el río Negro, con base en el índice biológico BMWP-Col, y el índice ecológico ICE $_{\mathrm{RN}-\mathrm{MAE}}$

TABLE 6

Classification of ecological state of the sampling points stations on the Negro river watershed based on BMWP-Col biological index and $\mathrm{ICE}_{\mathrm{RN}-\mathrm{MAE}}$ ecological index

\begin{tabular}{cclcl} 
Estación & BMWP-Col & Estado & ICE $_{\text {RN-MAE }}$ & Estado \\
E1 & 35 & Crítica & 5.6 & Regular \\
E2 & 38 & Dudosa & 6.1 & Buena \\
E3 & 50 & Dudosa & 6.4 & Buena \\
E4 & 134 & Buena & 7.9 & Buena \\
E5 & 99 & Aceptable & 6.9 & Buena \\
E6 & 19 & Crítica & 5.0 & Regular \\
E7 & 18 & Crítica & 5.4 & Buena \\
E8 & 69 & Aceptable & 7.4 & Regular \\
E9 & 17 & Crítica & 5.5 & Regular \\
E10 & 31 & Crítica & 5.6 & Buena \\
E11 & 59 & Dudosa & 8.1 & Buena \\
E12 & 8 & Muy crítica & 3.4 & Crítica \\
E13 & 43 & Dudosa & 7.6 & Buena \\
E14 & 22 & Crítica & 4.8 & Buena \\
E15 & 95 & Aceptable & 7.1 & Buena \\
E16 & 96 & Aceptable & 7.5 & Regular \\
E17 & 102 & Buena & 7.1 & Buena \\
E18 & 24 & Crítica & 5.4 & 6.9 \\
E19 & 44 & Dudosa & & \\
\hline
\end{tabular}


el gradiente de variación ambiental obtenido a partir del ACC comprende un amplio espectro de condiciones de calidad de agua, al cual se asocian variables que muestran un alto grado de dependencia que incluye la producción, el consumo y la descomposición.

Los resultados obtenidos muestran que oligoquetos como Erpobdellidae y Helobdella, junto con larvas de Chironomus, son los organismos más tolerantes a la contaminación generada por el enriquecimiento de materia orgánica e inorgánica en los sitios estudiados. Chalar et al. (2011) encontraron en la cuenca del río Santa Lucia (Uruguay), puntajes similares para estos organismos cuya capacidad de adaptación se manifiesta en que poseen hemoglobina y circulación forzada del agua en sus galerías, lo cual les permite resistir concentraciones muy bajas de oxígeno y alcanzar densidades poblacionales extremadamente altas (Roldán, 2003). No obstante, taxones como Biomphalaria (Mollusca) y Rhinoaeschna (Odonata) en la cuenca del río Negro, parecen ser más sensibles a la contaminación que en la cuenca estudiada por Chalar et al. (2011). Se establecieron para ellos, en esta investigación, valores óptimos y de tolerancia más cercanos a condiciones de buena calidad. Lo anterior resalta la necesidad de identificar, para cada región biogeográfica, los valores de óptimo y de tolerancia generados desde el estudio directo de los factores ambientales y de la distribución de los taxones.

ICE $_{\text {RN-MAE }}$ : El método empleado para la estimación del índice de calidad ecológica del río Negro combinó un análisis de gradiente directo (ACC) junto con un modelo de Promedios Ponderados (WA). El ACC es una técnica frecuentemente usada tanto para detectar gradientes ambientales como para ordenar los sitios a lo largo de ellos, mientras que el WA se utiliza para estimar el óptimo y la tolerancia de las especies en estudios ecológicos y paleolimnológicos; ambos análisis han sido propuestos como métodos efectivos y objetivos para el desarrollo de índices biológicos que evalúan la calidad ambiental (Ter Braak, 1987). En el caso de Colombia, no se hallaron publicaciones que muestren el empleo del ACC junto con análisis de WA para establecer puntajes óptimos y de tolerancia de macroinvertebrados. El único estudio en este país acerca del establecimiento de valores de bioindicación de macroinvertebrados, basado en las abundancias y en tolerancias mínimas de los géneros, se realizó mediante un análisis de factores (Riss et al., 2002a; 2002b).

El ICE $\mathrm{RN}_{\mathrm{RNAE}}$ es una forma numérica que expresa la posición de cada una de las estaciones de muestreo en un gradiente ambiental de calidad del agua. Es un índice que a diferencia del BMWP-Col (el cual ha sido establecido para Colombia mediante datos de presencia-ausencia), considera la abundancia de cada taxón. La importancia de usar datos de abundancia radica en su utilidad para definir organismos bioindicadores. Roldán (2003) menciona que un organismo es un buen indicador de variaciones espaciotemporales en la calidad del agua cuando se encuentra invariablemente en un ecosistema de características definidas y cuando su población es porcentualmente superior o ligeramente similar a la del resto de los organismos con los que comparte el mismo hábitat. De ahí que, generalmente, un cambio en la abundancia sea la respuesta más frecuentemente observada en las especies de macroinvertebrados ante los cambios en los niveles de nutrientes (Hart \& Robinson, 1990; Perrin \& Richardson, 1997; Stevenson, 1997). Es por eso que se precisa estimar no solo la composición de su comunidad sino también la abundancia de cada taxón como parte integral en la elaboración de cualquier índice biológico. De esta manera, el índice obtenido brinda una respuesta más objetiva y rigurosa para evaluar la calidad ecológica de un sistema.

En cuanto al nivel taxonómico que se recomienda utilizar en la elaboración de índices biológicos, es cierto que se necesita incrementar la resolución taxonómica (por ejemplo, de familia a especie). Esto debido a que el valor de tolerancia de cada familia representa en realidad un valor medio de los valores de tolerancia de los géneros o especies dentro de la familia, cuando de hecho cada uno de ellos 
requiere, por ejemplo, niveles diferentes de oxígeno u ocupar diferentes microhábitats lo cual puede reflejarse en puntaciones distintas (Furse, Moss, Wrigth \& Armitage, 1984; Haase $\&$ Nolte, 2008). En el Cuadro 3 se muestra un ejemplo de dicha situación, pues las puntuaciones óptimas están variando dentro de una misma familia.

Un caso particular y ampliamente discutido es el de la clasificación de los organismos de Chironomidae hasta el nivel de familia, con la consecuente utilización de dicha familia como indicadora de aguas fuertemente contaminadas (Alba-Tercedor, 1996; Riss et al., 2002b; Roldán, 2003), lo cual ha sido considerado erróneo en varios casos (McGill, Wilson \& Brake, 1979; Puntí, Rieradevall \& Prat, 2009; Al-Shami, Rawi, Hassan Ahmad \& Nor, 2010). En este trabajo se constató que ciertamente los integrantes de Chironomidae colectados abarcaron toda una gama de condiciones ecológicas, Chironomus tuvo una puntuación óptima de 4.4, que indica condiciones críticas; Polypedilum y Orthocladiinae morfoespecie 1, mostraron puntuaciones asociadas a condiciones regulares; mientras que Parachironomus, Endotribelos y Paratanytarsus entre otros, presentaron puntuaciones indicadoras de buen estado ecológico.

ICE $_{\text {RN-MAE }}$ y BMWP-Col: Estos son índices biológicos en los que se emplea a la comunidad de macroinvertebrados para evaluar la calidad del agua, y en donde a cada taxón se asigna una puntuación de acuerdo con la tolerancia que presente a la contaminación orgánica. No obstante, mientras que el BMWP-Col usa un enfoque cualitativo (presencia/ausencia) $\mathrm{y}$ un nivel taxonómico de familia, el $\mathrm{ICE}_{\mathrm{RN}-\mathrm{MAE}}$ es un índice cuantitativo, basado en correlaciones entre la abundancia de los taxones con variables ambientales para establecer niveles de tolerancia, y emplea un nivel taxonómico más refinado al trabajar con géneros o morfotipos.

Así, la alta correlación hallada entre los dos índices significa que la respuesta de ambos es similar en cuanto a la representación de la variabilidad de la calidad del agua presente en la cuenca del río estudiado. Sin embargo, consideramos que la diferencia entre ellos radica en: 1) la mayor capacidad discriminativa de las categorías establecidas con el ICE $_{\text {RN-MAE, }}$ tal como puede observarse en el Cuadro $6 ; 2$ ) es una regla de modelación que de dos índices referidos a un mismo fenómeno, es más consistente aquel que establezca un mayor número de categorías, como en este caso lo hace el $\mathrm{ICE}_{\mathrm{RN}}$ MAE; y 3) el empleo de índices que involucren resolución taxonómica a los niveles de género y/o especie es definitivamente aconsejable ya que es más precisa la respuesta de un género o la de una especie ante las perturbaciones ambientales que la de una familia u otra categoría taxonómica superior; por tanto, el $\mathrm{ICE}_{\mathrm{RN}}$ MAE muestra ser una avance en esta dirección.

Hurford, Schneider \& Cowx (2010) discute como el BMWP, y sus diferentes versiones, utilizan puntajes de las familias que son sometidos a un proceso de clasificación altamente subjetivo, esto es, según se mencionó, el criterio de expertos. Hulford et al. (2010) consideran que aunque la mayoría de los métodos pueden operar a nivel de familia, el análisis al nivel de género o especie permite un mayor grado de precisión, siendo la aproximación ideal la del nivel de especie, a pesar de los requerimientos que exige (mayor tiempo de identificación y buenos taxónomos). Sin embargo, los taxones que requieren un esfuerzo notable de identificación (disección, por ejemplo) para ser clasificados hasta especie pueden ser dejados al nivel de género o grupos de especies. Finalmente, los datos cualitativos obtenidos con el BMWP-Col están sujetos a variación observacional como producto de su subjetividad, mientras que los datos cuantitativos del $\mathrm{ICE}_{\mathrm{RN}-\mathrm{MAE}}$ son más objetivos y permiten, si así se desea, la comparación empírica de hábitats en varias escalas espaciales, generalmente en aquellas más pequeñas.

El $\mathrm{ICE}_{\mathrm{RN}-\mathrm{MAE}}$ es también una herramienta que posibilita determinar no solo el estado biológico de un sistema sino que además funciona para establecer su estado ecológico, pues contempla la identificación de un gradiente ambiental y correlaciones entre 
variables fisicoquímicas con la abundancia de los macroinvertebrados, condición que no cumple el BMWP-Col.

\section{AGRADECIMIENTOS}

Este trabajo contó con la financiación de la Corporación Autónoma Regional para los ríos Negro-Nare-CORNARE y del Comité para el Desarrollo de la Investigación de la Universidad de Antioquia (CODI). Agradecemos al grupo LimnoBasE-BiotaMAR de la Universidad de Antioquia, en especial a Fernando Muñoz, a Rodulfo Ospina de la Universidad Nacional de Colombia y a los evaluadores por sus contribuciones para el mejoramiento del manuscrito.

\section{RESUMEN}

Los índices disponibles para la evaluación del estado ecológico de los ríos en Colombia están basados principalmente en hipótesis subjetivas generalizadas acerca de la toleración de los macroinvertebrados, un proceso que presenta limitaciones. En este estudio se presenta la aplicación de un método para establecer un índice de calidad ecológica para sistemas lóticos, basado en abundancia de macroinvertebrados y variables fisicoquímicas. Se tomó como caso de estudio la cuenca del rio Negro (Colombia). Este nuevo índice se ha formulado como alternativa al BMWP-Col y consiste en la determinación de un gradiente ambiental a partir de un Análisis de Correspondencia Canónica de correlaciones entre abundancias y registros fisicoquímicos. Los puntajes de las estaciones -obtenidos en las correlaciones- son estandarizados y usados en un modelo de Promedios Ponderados (WA); en el modelo se ponderan también las abundancias para estimar los valores óptimos y de tolerancia de cada taxón. Con ellos y la abundancia se calcula los valores del Índice de Calidad Ecológica con base en macroinvertebrados acuáticos para la cuenca del rio Negro ( $\mathrm{ICE}_{\mathrm{RN}-\mathrm{MAE}}$ ). Se utilizan los valores del índice junto con las concentraciones de fósforo total (FT) en un análisis de agrupamiento, para así clasificar los sitios de estudio. De ahí se obtienen finalmente los valores medios, máximos, mínimos y de desviación estándar del $\mathrm{ICE}_{\mathrm{RN}-\mathrm{MAE}}$ y FT. Con ellos se definen los umbrales que corresponden a diferentes categorías de calidad ecológica (buena, regular y crítica).

Palabras clave: índice de calidad ecológica, BMWPCol, macroinvertebrados, gradiente ambiental, quebradas tropicales.

\section{REFERENCIAS}

Acosta, A. J. (2005). Evaluación de la calidad de agua de las microcuencas El cerro, La Madera, y La Chapa del municipio del Carmen de Viboral, departamento de Antioquia, utilizando macroinvertebrados acuáticos como indicadores biológicos. Rionegro, Colombia: Universidad Católica de Oriente.

Acosta, R., Ríos, B., Rieradevall, M. \& Prat, N. (2009). Propuesta de un protocolo de evaluación de la calidad ecológica de ríos andinos (CERA) y su aplicación a dos cuencas en Ecuador y Perú. Limnetica, 28, 35-64.

Al-Shami, S. A., Rawi, C. S. M., Hassan Ahmad, A. \& Nor, S. A. M. (2010). Distribution of Chironomidae (Insecta: Diptera) in polluted rivers of the Juru River Basin, Penang, Malaysia. Journal of Environmental Sciences, 22, 1718-1727.

Alba-Tercedor, J. \& Sánchez-Ortega, A. (1988). Un método rápido y simple para evaluar la calidad biológica de las aguas corrientes basada en el de Hellawell (1978). Limnética, 4, 51-56.

Alba-Tercedor, J. (1996). Macroinvertebrados acuáticos y calidad de las aguas de los ríos. IV Simposio del Agua en Andalucía (SIAGA), Almería, II, 203-213.

Arango, M. C., \& Roldán, G. (1983). Odonatos inmaduros del departamento de Antioquia en diferentes pisos altitudinales. Actualidades Biológicas, 12, 91-105.

Aristizábal, H. (2002). Los hemípteros de la película superficial del agua en Colombia. Bogotá, Colombia: Academia Colombiana de Ciencias Exactas, Físicas y Naturales.

Armitage, P. D., Moss, D., Wrigth, J. F. \& Furse, M. T. (1983). The performance of a new biological water quality score system based on macroinvertebrates over a wide range of unpolluted running-water sites. Water Research, 17, 333-347.

Bakkes, J. (1994). An Overview of Environmental Indicators: State of the Art and Perspectives. Nairobi, Kenia: Environmetal Assessment Sub Programme, UNEP.

Bejarano, E. E. (2006). Lista actualizada de los psicódidos (Diptera: Psychodidae) de Colombia. Folia Entomológica Mexicana, 45(001), 47-56.

Bispo, P. D. C., Froehlich, C. G., \& Oliveira, L. G. (2002). Stonefly (Plecoptera) fauna of streams in a mountainous area of Central Brazil: abiotic factors and nymph density. Revista Brasileira de Zoología, 19, 325-334.

Bonada, N., Zamora-Muñoz, C., Rieradevall, M., \& Prat, N. (2004). Ecological profiles of caddisfly larvae in Mediterranean streams: implications for bioassessment methods. Environmental pollution, 132, 509-21.

Borkent, A. \& Spinelli, G. (2007). Neotropical Ceratopogonidae, Diptera, Insecta (Aquatic Biodiversity in Latin America). Sofía, Bulgaria: Pensoft. 
Carvalho, C. (2002). Muscidae (Diptera) of the Neotropical Region: Taxonomy. Curitiba, Brasil: Universidade Federal do Paraná.

Chalar, G., Arocena, R., Pacheco, J. P., \& Fabián, D. (2011). Trophic assessment of streams in Uruguay: A Trophic State Index for Benthic Invertebrates (TSIBI). Ecological Indicators, 11, 362-369.

Diamond, J. M., Barbour, M. T., \& Stribling, J. B. (1996). Characterizing and comparing bioassessment methods and their results: a perspective. Journal of the North American Benthological Society, 15, 713-727.

Domínguez, E., \& Fernández, H. R. (2009). Macroinvertebrados Bentónicos Sudamericanos-Sistemática y Biología. Tucumán, Argentina: Fundación Miguel Lillo.

Domínguez, E., Molineri, C., Pescador, M., Hubbard, M. D., \& Nieto, C. (2006). Ephemeroptera of South America. Bulgaria: Pensoft.

Dudgeon, D. (2008). Tropical Stream Ecology (Aquatic Ecology). Lawrence, Kansas: Academic Press.

Epler, J. 2001. Identification manual for the larval Chironomidae (Diptera) of North and South Carolina. Special Publication SJ2001-SP13. Crawfordville, FL: EPA.

Esteves, F. de A. 2011. Fundamentos de Limnologia (3rd ed.). São Paulo State, Brazil: Interciência.

Furse, M. T., Moss, D., Wrigth, J. F., \& Armitage, P. D. (1984). The influence of seasonal and taxonomic factors on the ordination and classification of runningwater sites in Great Britain and on the prediction of their macro-invertebrate communities. Freshwater Biology, 14, 257-280.

Gaviria, A. (2000). Municipios de mi tierra: el camino para conocer nuestro departamento. Medellín, Colombia: El Mundo.

Guisande-González, C., Barreiro-Felpeto, A., ManeiroEstraviz, I., Riveiro-Alarcón, I., Vergara-Castaño, A., \& Vaamonde-Liste, A. (2006). Tratamiento de Datos. Galicia, España: Ediciones Díaz de Santos.

Gutiérrez, J. D., Riss, W., \& Ospina, R. (2004). Lógica difusa como herramienta para la bioindicación de la calidad del agua con macroinvertebrados acuáticos en la Sabana de Bogotá-Colombia. Caldasia, 26(1), 161-172.

Haase, R., \& Nolte, U. (2008). The invertebrate species index (ISI) for streams in southeast Queensland, Australia. Ecological Indicators, 8, 599-613.

Hart, D. D. (1994). Building a Stronger Partnership between Ecological Research and Biological Monitoring. Journal of the North American Benthological Society, 13, 110-116.

Hart, D. D., \& Robinson, C. (1990). Resource limitation in a stream community: phosphorus enrichment effects on periphyton and grazers. Ecology, 71, 1494-1502.
Heckman, C. W. (2006). Encyclopedia of South American Aquatic Insects: Odonata-Anisoptera. Dordrecht, Netherlands: Springer.

Heckman, C. W. (2008). Encyclopedia of South American Aquatic Insects: Odonata-Zygoptera: Illustrated Keys to Known Families, Genera, and Species in South America. Dordrecht, Netherlands: Springer.

Hellawell, J. (1989). Biological indicators of freshwater pollution and environmental management. London, UK: Elsevier.

Holdridge, L. (1982). Ecología Basada en Zonas de Vida. San José, Costa Rica: IICA.

Hurford, C., Schneider, M., \& Cowx, I. (2010). Conservation Monitoring in freshwater habitats. A practical guide and case studies. Nueva York, USA: Springer.

Kelly, M. G., \& Whitton, B. A. (1998). Biological monitoring of eutrophication in rivers. Hydrobiologia, 384, 55-67.

Machado, T., \& Ramírez, J. J. (2003). Los macroinvertebrados acuáticos y su relación con los cambios en las variables fisicas y químicas como indicadores de la calidad ecológica de la cuenca del río Negro (Informe final). Rionegro, Colombia: Corporación Autónoma Regional para los ríos Negro-Nare (Cornare).

Martínez, C. (2005). Introducción a Los Escarabajos Carabidae (Coleoptera) de Colombia. Bogotá, Colombia: Instituto de Investigación de Recursos Biológicos Alexander Von Humboldt.

McGill, J. D., Wilson, R. S., \& Brake, A. M. (1979). The use of Chironomid pupal exuviae in the surveillance of sewage pollution within a drainage system. Water Research, 13, 887-894.

Medina, C., Hora, M., Pereda, W., Aguilar, R., \& Guzmán, I. (2010). El indice Biological Monitoring Working Party (BMWP), modificado y adaptado a tres microcuencas del Alto Chicama, La Libertad, Perú. SCIÉNDO, 13, 5-20.

Milligan, M. R. (1997). Identification manual for the aquatic Oligochaeta of Florida. Florida, USA: State of Florida, Dept. of Environmental Protection, Division of Water Facilities.

MINAE-SALUD. (2007). Reglamento para la evaluación y clasificación de la calidad de cuerpos de agua superficiales. Decreto, No. 33903, La Gaceta No. 178. San José, Costa Rica.

Moya, N., Tomanova, S., \& Oberdorff, T. (2007). Initial development of a multi-metric index based on aquatic macroinvertebrates to assess streams condition in the Upper Isiboro-Sécure Basin, Bolivian Amazon. Hydrobiologia, 589, 107-116.

Muñoz-Riveaux, S., Naranjo-López, C., Garcés-González, G., González Lazo, D. D., Musle-Cordero, Y., \& Rodríguez-Montoya, L. (2003). Evaluación de la calidad del agua utilizando los macroinvertebrados bentónicos como bioindicadores. Revista Chapingo Serie Ciencias Forestales y del Ambiente, 9, 147-153. 
Navarrete-Heredia, J. L., Newton, A. F., \& Thayer, M. K. (2002). Guía ilustrada para los géneros de Staphylinidae (Coleoptera) de México. Guadalajara, México: Universidad de Guadalajara.

Perrin, C. J., \& Richardson, J. S. (1997). N and P limitation of benthos abundance in the Nechako River, British Columbia. Canadian Journal of Fisheries and Aquatic Sciences, 54(11), 2574-2583.

Prat, N., Gonzáles, G., \& Millet, X. (1986). Comparación crítica de dos índices de calidad del agua: ISQA y BILL. Tecnología del Agua, 31, 33-49.

Puntí, T., Rieradevall, M., \& Prat, N. (2009). Environmental factors, spatial variation, and specific requirements of Chironomidae in Mediterranean reference streams. Journal of the North American Benthological Society, 28, 247-265.

Resh, V. H., \& Unzicker, J. D. (1975). Water and aquatic quality monitoring of the importance organisms: identification species. Journal of the Water Pollution Control Federation, 47(1), 9-19.

Resh, V. H., Bêche, L. A., \& McElravy, E. P. (2005). How common are rare taxa in long-term benthic macroinvertebrate surveys? Journal of the North American Benthological Society, 24, 976-989.

Reynoldson, T., Norris, R., Resh, V., Day, K., \& Rosenberg, D. (1997). The reference condition: A comparison of multimetric and multivariate approaches to assess water-quality impairment using benthic macroinvertebrates. Journal of the North American Benthological Society, 16, 833-852.

Riss, W., Ospina, R., \& Gutiérrez, J. D. (2002a). Una metodología para el cálculo de valores primarios de bioindicación. Acta Biológica Colombiana, 7(2), 29-35.

Riss, W., Ospina, R., \& Gutiérrez, J. D. (2002b). Establecimiento de valores de bioindicación para macroinvertebrados acuáticos de la Sabana de Bogotá. Caldasia, 24, 135-156.

Roldán, G. (1980). Estudios limnológicos de cuatro ecosistemas neotropicales diferentes con espcial referencia a su fauna de efemerópteros. Actualidades Biológicas, 9(34), 103-117.

Roldán, G. (1988). Guía para el estudio de los macroinvertebrados acuáticos del Departamento de Antioquia. Medellín, Colombia: Fondo para la Protección del Medio Ambiente "José Celestino Mutis."

Roldán, G. (2003). Bioindicación de la calidad del agua en Colombia: Propuesta para el uso del método BMWPCol. Medellín, Colombia: Universidad de Antioquia.

Roldán, G., \& Ramírez, J. J. (2008). Fundamentos de Limnología Neotropical (2da ed.). Medellín, Colombia: Universidad de Antioquia.

Roldán, G., \& Pérez, G. (1978). Niveles de contaminación por detergentes y su influencia en las comunidades bénticas del río Negro. Actualidades Biológicas, $7(24), 27-36$
Rosenberg, D. M., \& Resh, V. H. (1993). Freshwater biomonitoring and benthic macroinvertebrates. London, UK: Chapman \& Hall.

Schuh, R., \& Slater, J. (1995). True bugs of the world (Hemiptera: Heteroptera): classification and natural history. European Journal of Entomology, 93(2), 336.

Smith, K. (1989). Handbooks for the Identification of British Insects: Diptera: An Introduction to the Immature Stages of British Flies. London, UK: Royal Entomological Society.

Springer, M., Vásquez, D., Castro, A., \& Kohlmann B. (2007). Bioindicadores de la calidad del agua. Guía de campo. Guácimo, Costa Rica: Universidad EARTH.

Stevenson, R. (1997). Resource Thresholds and Stream Ecosystem Sustainability. Journal of the North American Benthological Society, 16(2), 410-424.

Tejerina, E. G., \& Paggi, A. C. (2010). Biodiversidad de la familia Chironomidae (Diptera) en el Noroeste Argentino. Tucumán, Argentina: Universidad Nacional de Tucumán.

Ter Braak, C. J. F. (1987). Unimodal models to relate species to environment. Wageningen, Netherlands: Agricultural Mathematics Group.

Ter Braak, C. J. F., \& Smilauer, P. (2002). CANOCO for Windows Version 4.5 Centre for Biometry Wageningen CPRO-DLO. Wageningen, The Netherlands: Wageningen University.

Tomanova, S., \& Tedesco, P. A. (2007). Tamaño corporal, tolerancia ecológica y potencial de bioindicación de la calidad del agua de Anacroneuria spp (Plecoptera: Perlidae) en América del Sur. Revista de Biología Tropical, 55(1), 67-81.

Toro, G. (1979). Suelos del Departamento de Antioquia, (Tomo I). Bogotá, Colombia: Instituto Geográfico Agustín Codazzi.

Wiggins, G. B. (1996). Larvae of the North American caddisfly genera (Trichoptera) (2nd ed.). Toronto, Canadá: University of Toronto Press, Scholarly Publishing Division.

Zamora, H. (1999). Adaptación del índice BMWP para la evaluación biológica de la calidad de las aguas epicontinentales en Colombia. Revista Unicauca Ciencia, 4, 47-60.

Zamora-Muñoz, C., \& Alba-Tercedor, J. (1996). Bioassessment of organically polluted Spanish rivers, using a biotic index and multivariate methods. Journal of the North American Benthological Society, 15, 332-352.

Zúñiga, M. del C., \& Cardona, W. (2009). Bioindicadores de calidad de agua y caudal ambiental. En J. Cantera, \& Y. Carvajal, L. Castro, L. (Eds.), Caudal ambiental: conceptos experiencias y desafios (pp. 167-198). Cali, Colombia: Programa Editorial de la Universidad del Valle. 
